\title{
SPECTRA FOR A PAIR OF SINGULAR FIRST ORDER DIFFERENTIAL EQUATIONS
}

\author{
B. W. ROOS AND W. C. SANGREN
}

1. Introduction. It has been shown by Titchmarsh [1] how the classical methods of the theory of complex functions can be used to obtain the spectra and associated expansion theorems for the singular cases of

$$
x^{\prime \prime}(t)+[\lambda-q(t)] x(t)=0 \quad\left[{ }^{\prime}=d / d t\right] .
$$

Conte and Sangren [2] have shown how these results can be generalized to a particular singular case for the pair of first order equations

$$
\begin{aligned}
& x_{1}^{\prime}(t)-\left\{\lambda+q_{1}(t)\right\} x_{2}(t)=0, \\
& x_{2}^{\prime}(t)+\left\{\lambda+q_{2}(t)\right\} x_{1}(t)=0 .
\end{aligned}
$$

It is the objective of this paper to obtain the spectra for many additional singular cases of this pair of equations.

To avoid excessive details the presentation here will be formal. The omitted details are contained in a General Atomic report [3].

The main results of this paper can be summarized in the following theorem:

Theorem. Consider the system (1) over the semi-infinite interval $(0, \infty)$ and under the boundary conditions

$$
\cos \beta x_{1}(0)+\sin \beta x_{2}(0)=0,
$$

where $\beta$ is a real constant. A solution of the system (1), (2) is a vector $x$ with components $x_{1}$ and $x_{2}$ having continuous first derivatives and satisfying this system. The values of $\lambda$ for which such solutions exist are contained in

(i) the continuous spectrum when $S(t), F(t)$ and $\alpha(t)$ are real;

(ii) the point spectrum when $S(t)$ and $\alpha(t)$ are imaginary while $F(t)$ is complex.

Here $\alpha(t), F(t)$ and $S(t)$ are functions of $q_{1}(t)$ and $q_{2}(t)$ which are defined by equations (3), (6) and (8) respectively.

2. Preliminaries. On the finite interval $(0, b)$ let

$$
\phi(t, \lambda)=\left[\phi_{1}(t, \lambda), \phi_{2}(t, \lambda)\right] ; \quad \theta(t, \lambda)=\left[\theta_{1}(t, \lambda), \theta_{2}(t, \lambda)\right]
$$

be two vector solutions of (1) that satisfy the conditions

Presented to the Society, April 23, 1960; received by the editors June 23, 1960. 


$$
\begin{aligned}
\phi_{1}(0) & =-\sin \beta, & \phi_{2}(0) & =\cos \beta, \\
\theta_{1}(0) & =-\cos \beta, & \theta_{2}(0) & =-\sin \beta .
\end{aligned}
$$

The Wronskian of $\phi$ and $\theta$ is defined by

$$
W_{t}(\phi, \theta)=\phi_{1}(t) \theta_{2}(t)-\phi_{2}(t) \theta_{1}(t) .
$$

Because $W_{t}(\phi, \theta)$ is independent of $t$ and $W_{0}(\phi, \theta)=1, W_{t}(\phi, \theta)=1$, and $\phi$ and $\theta$ are linearly independent solutions.

The general solution of (1) may be written as $\theta(t, \lambda)+l(\lambda) \phi(t, \lambda)$. It is known $[4 ; 5]$ that if this general solution satisfies Sturmian boundary conditions at $t=b$, the eigenvalues will be real, simple, discrete and extend from $\lambda=-\infty$ to $\lambda=+\infty$. The corresponding eigenfunctions are real functions of $t$. For the singular case the spectrum can be investigated by taking the limit of the general solution as $b \rightarrow \infty$.

As in the case of singular second order differential equations it can be shown by a limit-circle, or limit-point argument that for $\operatorname{Im} \lambda \neq 0$ system (1) will have a vector solution:

$$
\Psi(t, \lambda)=\theta(t, \lambda)+m(\lambda) \phi(t, \lambda)
$$

belonging to the class of square integrable functions, $L^{2}(0, \infty)$. The function $m(\lambda)$ depends upon the limit of circles in the complex $\lambda$-plane and for $t \rightarrow \infty$ is either a limit-point or a point on a limit-circle. In the limit-circle case all solutions are in the class $L^{2}(0, \infty)$. Furthermore, $m(\lambda)$ is analytic for $\operatorname{Im} \lambda \neq 0$ and $m(\bar{l})=\overline{m(l)}$.

The spectral distribution function is determined by the imaginary part of $m(\lambda)$ [1]. The values of this distribution function in turn determine the spectrum of the system (1).

3. A transformation of the basic equations. In order to deal with the cases where the absolute value of $q_{1}(t), q_{2}(t)$ or both become large at infinity the following transformation of system (1) is considered. The independent variable $t$ is replaced by

$$
\alpha(t)=\int_{0}^{t}\left\{\left[\lambda+q_{1}(s)\right]\left[\lambda+q_{2}(s)\right]\right\}^{1 / 2} d s .
$$

Formally this transformation gives

$$
\begin{aligned}
& \frac{d u_{1}}{d \alpha}=u_{2}, \\
& \frac{d u_{2}}{d \alpha}=-u_{1}+R\left(\lambda, q_{1}, q_{2}\right) u_{1},
\end{aligned}
$$


where the components of the vector $u(t)$ are defined by

$$
\begin{aligned}
& u_{1}(t)=F(t) x_{2}(t), \\
& u_{2}(t)=-F^{-1}(t) x_{1}(t)+G(t) x_{2}(t),
\end{aligned}
$$

and

$$
\begin{aligned}
F(t, \lambda) & =\left[\lambda+q_{1}(t)\right]^{1 / 4}\left[\lambda+q_{2}(t)\right]^{-1 / 4} \\
G(t, \lambda) & =\left(\alpha^{\prime}\right)^{-1} F^{-1}(t, \lambda), \\
R\left(\lambda, q_{1}, q_{2}\right) & =\left(\alpha^{\prime}\right)^{-1} G^{\prime}(t, \lambda) F^{-1}(t, \lambda) .
\end{aligned}
$$

The transformed equations (4) have a form similar to (1). In contrast to equations (1), however, the coefficients on the right-hand sides of equation (4) do not in general become large when $\lambda$ does or when $q_{1}(t)$ or $q_{2}(t)$ or both become large.

It can be verified directly that a solution of (4) satisfies the integral equations

$$
\begin{aligned}
U_{1}(t, \lambda)= & U_{2}(0) \sin \alpha(t)+U_{1}(0) \cos \alpha(t) \\
& +\int_{0}^{t} U_{1}(s) S(s) \sin [\alpha(s)-\alpha(t)] d s, \\
U_{2}(t, \lambda)= & -U_{1}(0) \sin \alpha(t)+U_{2}(0) \cos \alpha(t) \\
& +\int_{0}^{t} U_{1}(s) S(s) \cos [\alpha(s)-\alpha(t)] d s
\end{aligned}
$$

where

$$
S(t)=G^{\prime}(t) F^{-1}(t) .
$$

4. Order properties of $U(t, \lambda)$. In order to determine the spectrum, the order properties of the solution of equations (7) for large values of $t$ must be obtained. Before this can be accomplished the behavior of $\alpha(t)$ for $t \rightarrow \infty$ needs to be determined. The following nine cases are considered as $t \rightarrow \infty$ :

(1) $q_{1}(t) \rightarrow+\infty, q_{2}(t) \rightarrow-\infty$;

(2) $\quad q_{1}(t) \rightarrow-\infty, q_{2}(t) \rightarrow+\infty$;

(3) $q_{1}(t) \rightarrow+\infty, q_{2}(t) \rightarrow+\infty$;

(4) $q_{1}(t) \rightarrow-\infty, q_{2}(t) \rightarrow-\infty$;

(5) $q_{1}(t) \rightarrow+\infty, q_{2}(t) \rightarrow 0$;

(6) $q_{1}(t) \rightarrow 0, \quad q_{2}(t) \rightarrow+\infty$;

(7) $q_{1}(t) \rightarrow-\infty, q_{2}(t) \rightarrow 0$;

(8) $q_{1}(t) \rightarrow 0, \quad q_{2}(t) \rightarrow-\infty$;

(9) $q_{1}(t) \rightarrow Q_{1}, \quad q_{2}(t) \rightarrow Q_{2}$.

It is advantageous to assume further that in all the cases $q_{1}(t)$ and 
$q_{2}(t)$ are such that $S(t)$ is $L(0, \infty)$. Not all of those cases require separate discussion.

Assume temporarily that $\lambda$ is fixed and that $\operatorname{Im} \lambda>0$. The character of $\alpha(t)$, which depends on the behavior of $q_{1}(t)$ and $q_{2}(t)$ as $t \rightarrow \infty$, can be determined after choosing a suitable branch cut leading to the principal value of the square root function. It can easily be seen that either $\operatorname{Im} \alpha(t) \rightarrow+\infty$ or $\operatorname{Im} \alpha(t) \rightarrow-\infty$. Similarly, it is possible to determine the behavior of $F(t)$ and $S(t)$. When $\operatorname{Im} \lambda \rightarrow 0$ the only two different cases turn out to be

(i) $S(t), F(t)$ and $\alpha(t)$ are simultaneously real.

(ii) $S(t), \alpha(t)$ are imaginary, $F(t)$ is complex.

The order properties of the solution of equations (7) for $t \rightarrow \infty$, can now be determined. Consequently, consider the case when $\operatorname{Im} \alpha(t)$ $\rightarrow \infty$ and $e^{-i \alpha(t)} \rightarrow+\infty$. It follows immediately from equations (7) that

$$
\begin{aligned}
Y_{1}^{+}(t) \equiv & e^{i \alpha(t)} U_{1}(t)=U_{2}(0) e^{i \alpha(t)} \sin \alpha(t)+U_{1}(0) e^{i \alpha(t)} \cos \alpha(t) \\
& +\int_{0}^{t} Y_{1}^{+}(s) S(s) e^{i[\alpha(t)-\alpha(s)]} \sin [\alpha(t)-\alpha(s)] d s \\
Y_{2}^{+}(t) \equiv & e^{i \alpha(t)} U_{2}(t)=-U_{1}(0) e^{i \alpha(t)} \sin \alpha(t)+U_{2}(0) e^{i \alpha(t)} \cos \alpha(t) \\
& +\int_{0}^{t} Y_{1}^{+}(s) S(s) e^{i[\alpha(t)-\alpha(s)]} \cos [\alpha(t)-\alpha(s)] d s .
\end{aligned}
$$

Now, since $\left|e^{i \alpha(t)} \sin \alpha(t)\right| \leqq 1$ and $\left|e^{i \alpha(t)} \cos \alpha(t)\right| \leqq 1$ it follows that

$$
\begin{aligned}
& \left|Y_{1}^{+}(t)\right| \leqq\left|U_{1}(0)\right|+\left|U_{0}(0)\right|+\int_{0}^{t}\left|Y_{1}^{+}(s) S(s)\right| d s, \\
& \left|Y_{2}^{+}(t)\right| \leqq\left|U_{1}(0)\right|+\left|U_{2}(0)\right|+\int_{0}^{t}\left|Y_{1}^{+}(s) S(s)\right| d s .
\end{aligned}
$$

The following lemma [2] can now be applied:

Lemma. Let $h_{1}, h_{2}, g_{1}, g_{2}$ be non-negative functions of $t$ over the interval $0 \leqq t \leqq t_{1}$; let $h_{1}, h_{2}$ be continuous and $g_{1}, g_{2}$ integrable over this interval. If $h_{1}, h_{2}$ satisfy the inequalities:

$$
h_{1}(t), h_{2}(t) \leqq M+\int_{0}^{t}\left[h_{1}(s) g_{1}(s)+h_{2}(s) g_{2}(s)\right] d s
$$

where $M$ is constant, then: 


$$
h_{1}(t), h_{2}(t) \leqq M \exp \left[\int_{0}^{t}\left\{g_{1}(s)+g_{2}(s)\right\} d s\right], \quad 0 \leqq t \leqq t_{1} .
$$

Consequently, if $S(t)$ is $L(0, \infty)$ then

$$
\begin{aligned}
& \left|Y_{1}(t)\right| \leqq\left[\left|U_{1}(0)\right|+\left|U_{2}(0)\right|\right] \exp \left[\int_{0}^{t}|S(s)| d s\right], \\
& \left|Y_{2}(t)\right| \leqq\left[\left|U_{1}(0)\right|+\left|U_{2}(0)\right|\right] \exp \left[\int_{0}^{t}|S(s)| d s\right] .
\end{aligned}
$$

$Y_{1}(t)$ and $Y_{2}(t)$ are, therefore, bounded for all $t$, if $S(t)$ is $L(0, \infty)$ and $\lambda$ is such that $U_{1}(0)$ and $U_{2}(0)$ are bounded. Hence, for large $t, U_{1}(t)$ $=O\left\{e^{-i \alpha(t)}\right\}$ and $U_{2}(t)=O\left\{e^{-i \alpha(t)}\right\}$.

For a fixed $\lambda$ in the upper half plane as $t \rightarrow \infty$ this gives

$$
\begin{aligned}
& U_{1}(t, \lambda)=e^{-i \alpha(t)}\left\{M^{+}(\lambda)+o(1)\right\}, \\
& U_{2}(t, \lambda)=e^{-i \alpha(t)}\left\{N^{+}(\lambda)+o(1)\right\},
\end{aligned}
$$

where

$$
\begin{aligned}
& M^{+}(\lambda)=-\frac{U_{2}(0)}{2 i}+\frac{U_{1}(0)}{2}-\frac{1}{2 i} \int_{0}^{\infty} e^{i \alpha(s)} S(s) U_{1}(s) d s, \\
& N^{+}(\lambda)=\frac{U_{1}(0)}{2 i}+\frac{U_{2}(0)}{2}+\frac{1}{2} \int_{0}^{\infty} e^{i \alpha(s)} S(s) U_{1}(s) d s .
\end{aligned}
$$

In a similar fashion when $\operatorname{Im} \alpha(t) \rightarrow-\infty$ and consequently $e^{i \alpha(t)}$ $\rightarrow \infty$ one can show that

$$
\begin{aligned}
& U_{1}(t, \lambda)=e^{i \alpha(t)}\left\{M^{-}(\lambda)+o(1)\right\}, \\
& U_{2}(t, \lambda)=e^{i \alpha(t)}\left\{N^{-}(\lambda)+o(1)\right\}
\end{aligned}
$$

where

$$
\begin{aligned}
& M^{-}(\lambda)=\frac{U_{2}(0)}{2 i}+\frac{U_{1}(0)}{2}+\frac{1}{2 i} \int_{0}^{\infty} e^{-i \alpha(s)} S(s) U_{1}(s) d s, \\
& N^{-}(\lambda)=-\frac{U_{1}(0)}{2 i}+\frac{U_{1}(0)}{2}+\frac{1}{2} \int_{0}^{\infty} e^{-i \alpha(s)} S(s) U_{1}(s) d s .
\end{aligned}
$$

5. The Wronskian of $\phi$ and $\theta$. From the basic transformation it follows that:

$$
U_{1}(0)=F(0) x_{2}(0) \text { and } U_{2}(0)=-F^{-1}(0) x_{1}(0)+G(0) x_{2}(0) .
$$


Furthermore, corresponding to the two linear independent solutions: $\phi(t, \lambda)$ and $\theta(t, \lambda)$ of the equation (1) one obtains

$$
\begin{array}{ll}
U_{1 \phi}(0)=F(0) \cos \beta, & U_{2 \phi}(0)=+F^{-1}(0) \sin \beta+G(0) \cos \beta, \\
U_{1 \theta}(0)=-F(0) \sin \beta, & U_{2 \theta}(0)=+F^{-1}(0) \cos \beta-G(0) \sin \beta .
\end{array}
$$

If $\lambda$ is assumed to be real then from equations (7) and relations (9) it follows that

$$
\begin{aligned}
& U_{1}(t, \lambda)=\mu(\lambda) \sin \alpha(t)+\nu(\lambda) \cos \alpha(t)+o(1), \\
& U_{2}(t, \lambda)=-\nu(\lambda) \sin \alpha(t)+\mu(\lambda) \cos \alpha(t)+o(1),
\end{aligned}
$$

where

$$
\begin{aligned}
& \mu(\lambda)=U_{2}(0)+\int_{0}^{\infty} U_{1}(s) S(s) \cos \alpha(s) d s, \\
& \nu(\lambda)=U_{1}(0)-\int_{0}^{\infty} U_{1}(s) S(s) \sin \alpha(s) d s .
\end{aligned}
$$

The integrals in these two relations converge uniformly in $\lambda$ and hence $\mu, \nu$ are continuous and bounded functions of $\lambda$. Again, it has been assumed that $S(t)$ is $L(0, \infty)$.

The Wronskian of $\phi$ and $\theta$ is given by

$$
W_{t}[\phi, \theta]=\phi_{1} \theta_{2}-\phi_{2} \theta_{1}=1 .
$$

From the basic transformation one obtains

$$
\begin{aligned}
W_{t}[\phi, \theta] & =U_{1 \phi} U_{2 \theta}-U_{2 \phi} U_{1 \theta} \\
& =\left(\mu_{\theta} \nu_{\phi}-\mu_{\phi} \nu_{\theta}\right)+o(1) .
\end{aligned}
$$

Consequently, when $t \rightarrow \infty$,

$$
\mu_{\theta} \nu_{\phi}-\mu_{\phi} \nu_{\theta}=1 .
$$

Also, it follows that neither $\mu_{\phi}, \nu_{\phi}$ nor $\mu_{\theta}, \nu_{\theta}$ can vanish simultaneously for the same $\lambda$.

6. The nature of the spectrum. It was noted in the preliminaries that the system (1) has a two component vector solution

$$
\Psi(t, \lambda)=\theta(t, \lambda)+m(\lambda) \phi(t, \lambda)
$$

which is $L^{2}(0, \infty)$ for values of $\lambda$ for which $\operatorname{Im} \lambda \neq 0$. The functions $\phi$ and $\theta$ separately do not belong to $L^{2}(0, \infty)$. The nonintegrability of $\phi$ and $\theta$ can be verified by observing that

$$
F^{-1}(t)=\left\{\lambda+q_{2}(t)\right\}^{1 / 4}\left\{\lambda+q_{1}(t)\right\}^{-1 / 4}
$$


cannot vanish in a manner which will compensate for the growth of the term $e^{-i \alpha(t)}$ when $\operatorname{Im} \alpha(t) \rightarrow+\infty$ (or of the term $e^{i \alpha(t)}$ when $\operatorname{Im} \alpha(t) \rightarrow-\infty)$.

When $\operatorname{Im} \alpha(t) \rightarrow+\infty$ it follows that

$$
\begin{aligned}
\Psi_{1}^{+} & =\theta_{1}^{+}+m^{+}(\lambda) \phi_{1}^{+} \\
& =e^{-i \alpha(t)}\left[G M_{\theta}^{+}-F N_{\theta}^{+}+m^{+}\left\{G M_{\phi}^{+}-F N_{\phi}^{+}\right\}+o(1)\right], \\
\Psi_{2}^{+} & =\theta_{2}^{+}+m^{+}(\lambda) \phi_{2}^{+}=e^{-i \alpha(t)}\left[F^{-1} M_{\theta}^{+}+m^{+} F^{+} M_{\phi}+o(1)\right] .
\end{aligned}
$$

If $\Psi$ is to be $L^{2}(0, \infty)$ it follows that

$$
m^{+}(\lambda)=-\frac{M_{\theta}^{+}(\lambda)}{M_{\phi}^{+}(\lambda)}=-\lim _{t \rightarrow \infty} \frac{G(t) M_{\theta}^{+}(\lambda)-F(t) N_{\theta}^{+}(\lambda)}{G(t) M_{\phi}^{+}(\lambda)-F(t) N_{\phi}^{+}(\lambda)} .
$$

These formulas are also suitable when $\operatorname{Im} \alpha(t) \rightarrow-\infty$ providing $m^{+}(\lambda)$ is replaced by $m^{-}(\lambda)$ and $M^{+}, N^{+}$, are replaced respectively by $M^{-}$, $N^{-}$.

In the situation where the condition (1) of $\$ 4$ applies for $t \geqq 0$ one has

$$
M_{\phi}^{+}(\lambda)=\frac{1}{2 i}\left[-U_{2 \phi}(0)+i U_{1 \phi}(0)-\int_{0}^{\infty} e^{i \alpha(s)} S(s) F(s) \phi_{2}(s) d t\right],
$$

where

$$
\begin{aligned}
& U_{2 \phi}(0)=F^{-1}(0) \sin \beta+G(0) \cos \beta \\
& U_{1 \phi}(0)=F(0) \cos \beta
\end{aligned}
$$

Consequently,

$$
M_{\phi}^{+}(\lambda)=-\frac{1}{2 i}\left[\mu_{\phi}(\lambda)-i \nu_{\phi}(\lambda)\right]
$$

In a similar fashion

$$
\begin{aligned}
& M_{\theta}^{+}(\lambda)=-\frac{1}{2 i}\left[\mu_{\theta}(\lambda)-i \nu_{\theta}(\lambda)\right], \\
& N_{\phi}^{+}(\lambda)=\frac{1}{2}\left[\mu_{\phi}(\lambda)-i \nu_{\phi}(\lambda)\right] \\
& N_{\theta}^{+}(\lambda)=\frac{1}{2}\left[\mu_{\phi}(\lambda)-i \nu_{\theta}(\lambda)\right] .
\end{aligned}
$$

Analogous expressions hold for $M^{-}$and $N^{-}$. Formally, therefore, 


$$
m^{+}(\lambda)=-\frac{\mu_{\theta} \mu_{\phi}+\nu_{\theta} \nu_{\phi}}{\mu_{\phi}^{2}+\nu_{\phi}^{2}}-\frac{i}{\mu_{\phi}^{2}+\nu_{\phi}^{2}} .
$$

Hence

$$
\operatorname{Im} m^{+}(\lambda)=-\frac{1}{\mu_{\phi}^{2}+\nu_{\phi}^{2}}
$$

and

$$
\operatorname{Im} m^{-}(\lambda)=\frac{1}{\mu_{\phi}^{2}+\nu_{\phi}^{2}},
$$

In both cases $\operatorname{Im} m^{+}(\lambda)$ and $\operatorname{Im} m^{-}(\lambda)$ are continuous, nonvanishing functions of $\lambda$. The spectral distribution function will be continuous in this case and the interval of the real $\lambda$-axis under consideration will be in the continuous spectrum.

Next, assume that condition (ii) is satisfied for $t \geqq 0$ and that $S(t)$ and $\alpha(t)$ are imaginary while $F(t)$ is complex. From the definition one has

$$
\begin{aligned}
M_{\phi}^{+}=\frac{1}{2 i}\left[-F^{-1}(0) \sin \beta-G(0) \cos \beta+\right. & i F(0) \cos \beta \\
& \left.-\int_{0}^{\infty} e^{i \alpha(s)} S(s) F(s) \phi_{2}(s) d s\right] .
\end{aligned}
$$

Now it is easily seen that $F(t)=e^{i \pi / 4}|F(t)|, G(t)=e^{-i \pi / 4}|G(t)|$. Consequently, $e^{-\pi i / 4} M_{\phi}^{+}$is real. In a similar fashion $M_{\theta^{+}}^{+}, N_{\phi}^{+}, N_{\theta^{+}}, M_{\bar{\phi}}^{-}, M_{\bar{\theta}}^{-}$, $N_{\bar{\phi}}, N_{\bar{\theta}}$ can be shown to be real when multiplied either by $e^{i \pi / 4}$ or $e^{-i \pi / 4}$. Therefore, $m^{+}(\lambda)$ and $m^{-}(\lambda)$ are meromorphic functions of $\lambda$ because they are the ratio of integral functions. The discontinuity points of the spectral distribution function are the poles of the meromorphic functions $m^{+}(\lambda)$ or $m^{-}(\lambda)$ and the interval of the real $\lambda$-axis under consideration will be in the point spectrum.

For a fixed real $\lambda$ and given $q_{1}(t)$ and $q_{2}(t)$ the functions $\alpha(t), F(t)$ and $S(t)$ may not satisfy conditions (i) or (ii) separately for all $t$. However, if $t$ is chosen larger than some $t_{L}, \alpha(t), F(t)$ and $S(t)$ will satisfy only one of the conditions (i) or (ii) for $t \geqq t_{L}$. The character of $m^{+}(\lambda)$ and $m^{-}(\lambda)$ is then determined in the previous fashion where, however, the formulas are altered appropriately.

For the cases listed in $\S 4$ the following may be noted:

Case (1) and (2) point spectrum $-\infty<\lambda<\infty$,

Case (3) and (4) continuous spectrum $-\infty<\lambda<\infty$, 
Case (5) and (6) continuous spectrum $0<\lambda<\infty$, point spectrum $-\infty<\lambda<0$,

Case (7) and (8) point spectrum $0<\lambda<\infty$, continuous spectrum $-\infty<\lambda<0$,

Case (9) point spectrum $Q_{1}<\lambda<Q_{2}$, continuous spectrum $\lambda<\min \left[Q_{1}, Q_{2}\right], \lambda>\max \left[Q_{1}, Q_{2}\right]$.

\section{REFERENCES}

1. E. C. Titchmarsh, Eigenfunction expansions associated with second order differential equations, Oxford, Clarendon Press, 1946.

2. S. D. Conte and W. C. Sangren, An expansion theorem for a pair of first order equations, Canad. J. Math. vol. 6 (1954) pp. 554-560.

3. B. W. Roos and W. C. Sangren, Spectra for a pair of first order differential equations, San Diego, California, General Atomic Report GA 1373, 1960.

4. E. A. Coddington and N. Levinson, Theory of ordinary differential equations, New York, McGraw-Hill, 1955.

5. W. Hurwitz, An expansion theorem for a pair of linear first order differential equations, Trans. Amer. Math. Soc. vol. 22 (1921) pp. 526-543.

John Jay Hopkins Laboratory for Pure and Applied Science, General Atomic Division, General Dynamics Corporation

\section{A METHOD OF APPROXIMATING THE ZEROS OF FUNCTIONS BY QUADRATIC FORMULAS ${ }^{1}$}

\section{STEPHEN KULIK}

1. Introduction. The problem of approximating two zeros of a given function by solving a quadratic equation was discussed in a number of papers $[1 ; 2 ; 4 ; 5 ; 7]$. In this paper we present a general method of deriving quadratic equations the two roots of which would approximate two zeros of an analytic function $f(z)$. A function $f(z) / g(z, u)$, instead of $f(z)$, is considered, where $g(z, u)$ is another appropriately chosen analytic function. By varying the parameter $u$, and keeping the initial approximation to the zeros unchanged, the final approximations can be improved or another pair of zeros approximated. The exact values of the zeros are determined as limits of the expressions approximating them.

2. The general method. Let $f(z)$ and $g(z, u)$ be analytic functions

Received by the editors September 7, 1959.

' Sponsored by the Office of Ordnance Research, U. S. Army. 